\title{
Gravidez pós cirurgia bariátrica: uma revisão integrativa
}

\author{
Pregnancy after bariatric Surgery: an integrative review \\ Embarazo después de la cirugía bariátrica: una revisión integradora
}

July Jenny Blas Rosales ${ }^{1 *}$, Luis Alberto Leon Camac², Jacinta Lima Rogério Cardoso ${ }^{1}$, Larissa Boaes Nascimento ${ }^{1}$, Alan Guillermo Avila Westry ${ }^{3}$

\section{RESUMO}

Objetivo: Pesquisar na literatura científica quais são os fatores de risco associados a gravidez pós cirurgia bariátrica, bem como seus benefícios e recomendações. Métodos: Foi realizada uma revisão bibliográfica nas bases de dados PUBMED, Science Direct, NCBI e Scielo, usando como critério de inclusão artigos originais disponíveis, publicados no período de 2015 a 2019, nos idiomas inglês e português, e como critérios de exclusão, artigos duplicados e aqueles sem relação com o tema. Resultados: Compõem essa pesquisa 12 artigos, nos quais foi possível averiguar que a gestação pós cirurgia bariátrica deve ser concebida no período entre 12 a 24 meses após o procedimento, com a finalidade de evitar complicações materno e fetal assim como menor tempo de duração da gestação, partos prematuros espontâneos, abortos, malformações congênitas. Considerações Finais: Mulheres que engravidam após cirurgia bariátrica apresentam menor risco de apresentar diabetes gestacional, hipertensão gestacional, macrossomia fetal e maior risco de apresentar deficiências nutricionais maternas assim como recém-nascido com baixo peso ao nascer, restrição de crescimento intrauterino, partos prematuros e mortalidade intrauterina e neonatal.

Palavras-chave: Cirurgia bariátrica, Gestação, Bem-estar materno.

\begin{abstract}
Objective: To search the scientific literature what are the risk factors associated with pregnancy after bariatric surgery, benefits and recommendations Methods: A bibliographic review was carried out in the PUBMED, Science Direct, NCBI and Scielo databases, using as inclusion criteria available original articles published in the period from 2015 to 2019, in the English and Portuguese, as exclusion criteria duplicate articles and those unrelated to theme. Results: This research consists of 12 articles in which it was found that pregnancy after bariatric surgery should be conceived within 12 to 24 months after the procedure, in order to avoid maternal and fetal complications as well as shorter duration of gestation, deliveries spontaneous premature infants, miscarriages, congenital malformations. Final Considerations: Women who become pregnant after bariatric surgery have a lower risk of gestational diabetes, gestational hypertension, fetal macrosomia and a higher risk of maternal nutritional deficiencies as well as low birth weight newborns, intrauterine growth restriction, premature births and intrauterine and neonatal mortality.
\end{abstract}

Keywords: Bariatric surgery, Pregnancy, Maternal weel - being.

\section{RESUMEN}

Objetivo: Revisar, en la literatura científica cuales son los factores de riesgo asociados con el embarazo después de la cirugía bariátrica, beneficios y recomendaciones Métodos: Se realizó una revisión bibliográfica

\footnotetext{
${ }^{1}$ Hospital universitário Getúlio Vargas (HUGV), Manaus - Amazonas. *E-mail: julyrosa09@gmail.com

2 Universidade Federal do Amazonas (UFAM), Manaus - Amazonas.

3 Universidade Federal de Mato Grosso (UFMT), Cuiabá- Mato Grosso.
} 
en las bases de datos PUBMED, Science Direct, NCBI y Scielo utilizando como criterio de inclusión los artículos originales publicados de 2015 a 2019, en inglés y portugués, y como criterio de exclusión artículos duplicados y aquellos no relacionados con el tema. Resultados: Fueron evaluados 12 artículos que componen esta investigación, em los cuales fue posible verificar que el embarazo después de la cirugía bariátrica debe concebirse dentro de los 12 a 24 meses posteriores al procedimiento, para evitar complicaciones maternas y fetales, menor tiempo de duración de la gestación, nacimientos prematuros espontáneos, abortos, malformaciones congénitas. Consideraciones finales: Las mujeres que quedan embarazadas después de la cirugía bariátrica tienen un menor riesgo de diabetes gestacional, hipertensión gestacional, macrosomia fetal y un mayor riesgo de deficiencias nutricionales maternas, así como recién nacidos de bajo peso al nacer, retardo de crecimiento intrauterino, partos prematuros y mortalidad intrauterina y neonatal.

Palabras clave: Cirugía bariátrica, Embarazo, Bienestar materno.

\section{INTRODUÇÃO}

É notório que nos últimos anos o aumento da prevalência da obesidade no mundo, sendo assim considerada como uma doença crônica, tornando - se um problema de saúde pública. Dados da Organização Mundial de Saúde (OMS) do ano de 2016 mostram que cerca de 1,9 bilhão de adultos, com 18 anos ou mais, são afetados pelo excesso de peso, com Índice de Massa Corpórea (IMC) variando entre $25-29 \mathrm{~kg} / \mathrm{m}^{2}$, dos quais $39 \%$ pertencem ao sexo masculino e $40 \%$ pertencem ao sexo feminino.

A obesidade está associada a inúmeras comorbidade, tais como hipertensão, distúrbios músculo esqueléticos, câncer, diabetes do tipo 2, infertilidade, e principalmente em mulheres, e complicações relacionadas a gestação e ao período gestacional (GONZÁLES I, et al., 2016; CUNHA CPV, 2018; SHAWE J, et al., 2019).

O manejo da obesidade é bastante complexo e de caráter individualizado, e leva em consideração as condições físicas, clínicas e psicológicas da paciente, envolvendo abordagens de controle variáveis, tais como processos nutricionais, físicos e, principalmente, aspectos cirúrgicos.

Dentre os aspectos cirúrgicos, a Cirurgia Bariátrica (CB) vem apresentando cada vez mais popularidade, e dentre os procedimentos mais comumente realizados em pacientes obesos temos o Bypass Gástrico em $Y$ de Roux (RYGB), a Banda Gástrica Ajustável (BGA) e a Gastrectomia Vertical (GV) por laparoscopia (SHAWE J, et al., 2019).

A CB surgiu como uma abordagem bastante promissora, a qual é indicada quando outras formas de perda de peso não apresentem sucesso ou em casos de impossibilidade do paciente em ser submetido as demais abordagens, sendo a forma mais eficaz de perda de peso a longo prazo. Tal procedimento deve ser considerado se o IMC do paciente exceder $40 \mathrm{~kg} / \mathrm{m}^{2}$ e/ou estar entre $35 \mathrm{~kg} / \mathrm{m}^{2}$ a $40 \mathrm{~kg} / \mathrm{m}^{2}$ no caso de o mesmo apresentar relatos de comorbidades graves associadas a obesidade.

A ocorrência da CB diminui a incidência de comorbidades e complicações relacionadas a obesidade, como diabetes do tipo 2 e hipertensão, assim como ocorrência de complicações neonatais em gestantes, como diabetes gestacional e hipertensão. Porém, deficiências nutricionais relacionadas a absorção de micronutrientes são elevadas nesses procedimentos, como distúrbios na homeostase neuro-endócrina e metabólica (GONZÁLEZ I, et al., 2016; SHAWE J, et al., 2019).

Atualmente, é perceptível um crescimento exponencial no número do procedimento de $\mathrm{CB}$, sendo aproximadamente $80 \%$ das cirurgias no mundo realizadas em mulheres, principalmente as que se encontram em idade reprodutiva, pois esta pode melhorar a fertilidade, promovendo a restauração da ovulação. Entretanto, devido à má absorção de micronutrientes e alterações morfológicas no estômago e órgãos adjacentes que os procedimentos induzem, existe um potencial maior para resultados perinatais adversos, tais como nascimento prematuro, anormalidades congênitas e mortalidade perinatal (KIZY S, et al., 2017). 
Assim como o crescimento no número de procedimentos de $\mathrm{CB}$, tem-se seguido de um aumento significativo de mulheres que engravidam após serem submetidas a CB. Com isso, a gravidez pós procedimento de CB pode aumentar risco de comorbidade e/ou mortalidade materna. Sendo então necessárias orientações clínicas e um manejo nutricional específicos para aquelas pacientes que desejam uma gestação, pós procedimento de CB.

Posto que a obesidade tornou -se um grande problema de saúde pública nos últimos anos e, simultaneamente o aumento da prevalência de realização de CB em mulheres em idade fértil, não tem-se, até o momento, consenso sobre a condução e tempo de espera para a gravidez após o procedimento de $\mathrm{CB}$ (BUSETTO L, et al., 2018)

Embora o procedimento pareça reduzir os problemas de fertilidade relacionados à obesidade e os resultados adversos da gravidez, é necessário considerar as complicações relacionadas à gravidez possivelmente causadas pela $\mathrm{CB}$, como deficiências nutricionais, anemias, baixos níveis proteicos e de vitaminas. Assim como o histórico de uma paciente de CB ter forte associação ao metabolismo alterado e disfuncional da glicose, o que pode afetar diagnósticos, especialmente de hiperglicemia (SHAWE J, et al., 2019).

As gestantes com histórico de procedimento de desvio gástrico encontram-se em um grupo de maior risco a desenvolver hérnias internas, as quais apresentam um potencial bastante elevado a desenvolver consequências muito graves em relação as mesmas, como por exemplo a necrose intestinal ou até mesmo perfuração aguda. Tais condições podem estimular partos cesáreos agudos e/ ou precoces ou até mesmo casos excepcionais com resultado e morte materna e fetal (FALCONE V, et al., 2018).

A formação dessas hérnias internas após o procedimento de CB ocorre devido ao fato de que o útero apresenta-se em tamanho maior, acarretando uma elevação do intestino, e assim aumentando a pressão intra-abdominal, assim como intensificando o risco de malformações fetais, risco de aborto espontâneo e morte fetal intrauterina. Dessa forma, o padrão alimentar dessas gestantes deve ser monitorado e acompanhado de forma assídua, a fim de evitar complicações ao feto, devido a desnutrição ou má alimentação materna (FALCONE V, et al., 2018).

Com base no exposto acima, é essencial o conhecimento das consequências da CB nos resultados maternos e fetais durante e após o período gestacional. Sendo assim, o objetivo desse trabalho foi realizar um levantamento bibliográfico sobre as principais informações relacionadas a gravidez pós CB identificando os fatores de risco e benefícios, bem como consequências da gestação pós $C B$, principais complicações e recomendações.

\section{MÉTODOS}

A metodologia utilizada foi de revisão integrativa de literatura, realizando buscas nas bases de dados $U$. S. National Library of Medicine National Institutes of Health (PUBMED), Nacional Center for Biotechnology (Science Direct, NCBI) e Scientific Electronic Library Online (Scielo).

Foram determinados como critérios de inclusão artigos originais disponíveis na íntegra, que tenham sido publicados no período entre 2015 a 2019, nos idiomas inglês e português, e como critérios de exclusão, artigos duplicados, sem relação com o tema e que não estivessem disponíveis na íntegra.

As buscas foram realizadas utilizando como descritores os seguintes termos: "Pregnancy after bariatric surgery", "Bariatric surgery and gestation" "Bariatric surgery and pregnancy" e "Complications in pregnancy bariatric surgery". Após realizada a análise dos artigos coletados nas bases de dados, foram encontrados 540 , dentre os quais 152 na base PUBMED, 257 na base Science Direct, 100 na base NCBI e 30 na base Scielo. Desse total, foi realizada uma análise baseada nos critérios de exclusão e inclusão.

Após realizado o processo de triagem, foram selecionados 100 artigos para avaliação de conteúdo, sendo após esta avaliação, se enquadraram dentro dos critérios de inclusão apenas 12 artigos, os quais compuseram essa pesquisa (Figura 1) 
Figura 1 - Fluxograma de seleção dos artigos que integram a pesquisa.
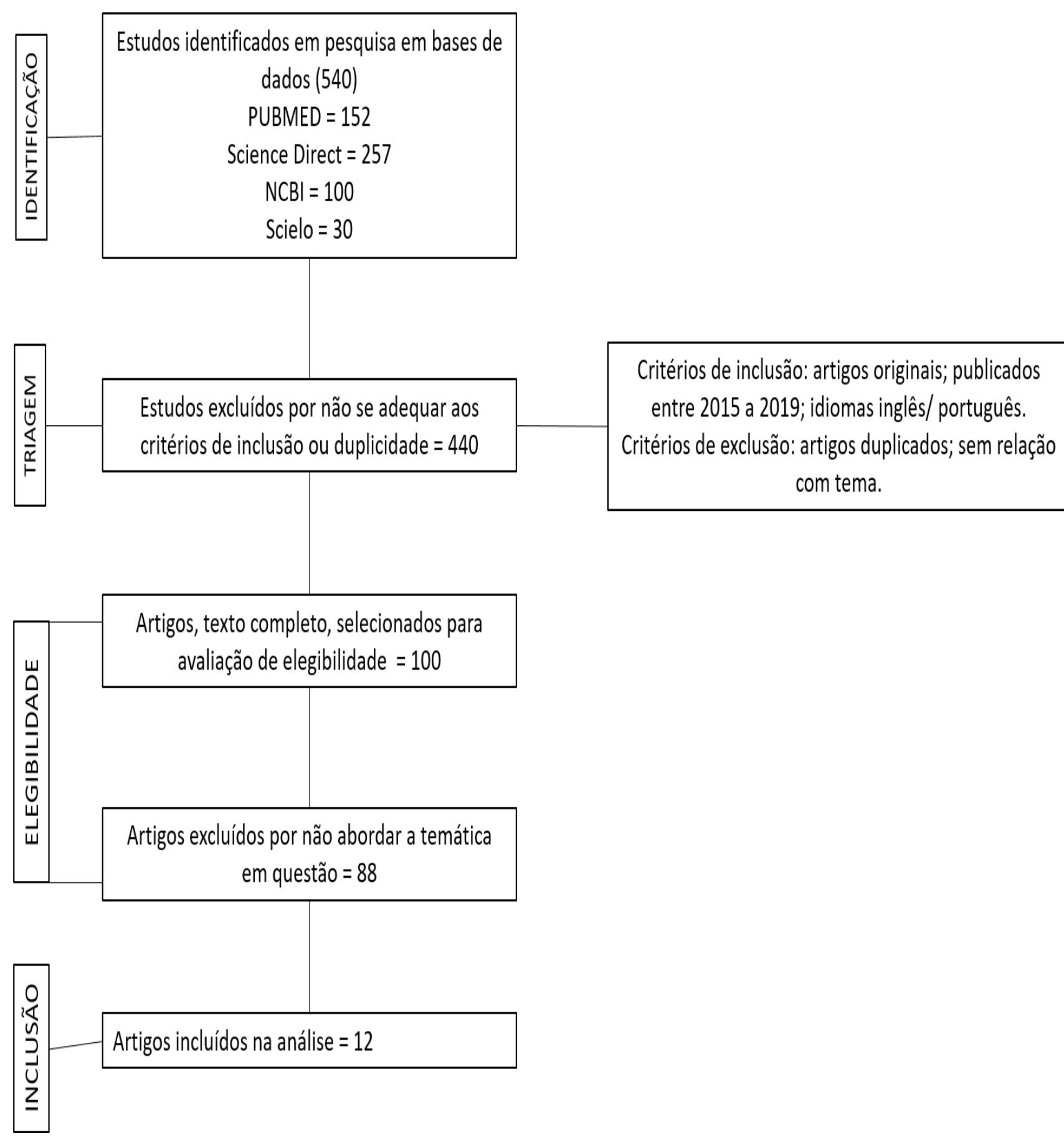

Fonte: Rosales JJB, et al., 2020.

\section{RESULTADOS}

Foram utilizados artigos que discorriam sobre gravidez pós o procedimento de Cirurgia Bariátrica, informando: 1) os risco e os benefícios da $\mathrm{CB}$; 2 ) as consequências da gestação pós procedimento de $\mathrm{CB}$ e 3 ) as complicações e as recomendações para gestações pós procedimento de CB. Dentre estes, foram encontrados 5 artigos na base Science Direct, 3 na base NCBI, 2 na base PUBMED e 2 na base Scielo, resultando em 12 artigos no total. Para melhor compreensão, os estudos foram organizados por descrição de algumas características, tais como: autores, ano de publicação, periódico, tipo de estudo, objetivos, interpretação do estudo e resultados, como pode ser observado no Quadro 1 
Quadro 1 - Caracterização de artigos incluídos na pesquisa. Manaus, Brasil 2018.

\begin{tabular}{|c|c|c|c|c|}
\hline Autor/ Ano & Periódico & $\begin{array}{l}\text { Tipo de } \\
\text { estudo }\end{array}$ & Objetivo & Interpretação do Estudo \\
\hline $\begin{array}{l}\text { Johansson } \\
\text { K, et al. } \\
\text { (2015) }\end{array}$ & Science Direct & Coorte & $\begin{array}{l}\text { Investigar risco de diabetes } \\
\text { gestacional e resultado } \\
\text { perinatais adversos em } \\
\text { mulheres pós CB em } \\
\text { relação a mulheres sem } \\
\text { histórico. }\end{array}$ & $\begin{array}{l}\text { Gestações após cirurgia bariátrica em comparação com gestações controle foram } \\
\text { associadas a menores riscos de diabetes gestacional. Porém foram associadas a maior } \\
\text { risco de partos prematuros, menor duração da gestação risco aumentado de natimorto ou } \\
\text { morte neonatal. }\end{array}$ \\
\hline $\begin{array}{l}\text { Parker MH, } \\
\text { et al. (2016) }\end{array}$ & $\mathrm{NCBI}$ & $\begin{array}{l}\text { Coorte } \\
\text { retrospect } \\
\text { ivo }\end{array}$ & $\begin{array}{l}\text { Avaliar se a } \text { CB está } \\
\text { associada à restrição de } \\
\text { crescimento intra-uterino } \\
\text { (RCIU). }\end{array}$ & $\begin{array}{l}\text { As gestações em mulheres obesas com CB prévia foram comparadas com não } \\
\text { obesas. Os desfechos foram pré-eclâmpsia, RCIU, morte fetal intra-uterina, grande para } \\
\text { a idade gestacional, hemorragia pós-parto, falha na indução do parto, parto vaginal e } \\
\text { cesárea. Foram identificadas } 186.605 \text { mulheres obesas com gestações únicas das quais } \\
1.585 \text { realizaram CB prévia, a qual foi associada a um risco aumentado de RCIU. }\end{array}$ \\
\hline $\begin{array}{l}\text { Kwong W, et } \\
\text { al. (2016) }\end{array}$ & PUBMED & $\begin{array}{l}\text { Meta- } \\
\text { análise }\end{array}$ & $\begin{array}{l}\text { Avaliar os benefícios e } \\
\text { riscos da CB em mulheres } \\
\text { obesas de acordo com os } \\
\text { resultados obstétricos. }\end{array}$ & $\begin{array}{l}\text { Embora a CB esteja associada a redução no risco de desfechos obstétricos adversos, } \\
\text { existe potencial para risco aumentado de outros desfechos como restrição do crescimento } \\
\text { intrauterino e pequeno para idade gestacional, relacionados a deficiências de } \\
\text { micronutrientes durante a gravidez após CB, como proteínas, vitaminas B, ácidos graxos, } \\
\text { zinco, ácido fólico. }\end{array}$ \\
\hline $\begin{array}{c}\text { Marques RR, } \\
\text { et al. (2016) }\end{array}$ & PUBMED & $\begin{array}{c}\text { Retrospec } \\
\text { tivo }\end{array}$ & $\begin{array}{l}\text { Relatar resultados } \\
\text { obstétricos e perinatais de } \\
\text { gestações ocorridas após } \\
\text { realização de CB. }\end{array}$ & $\begin{array}{l}\text { Análise de prontuários de pacientes do gênero feminino com idade inferior a } 50 \text { anos, } \\
\text { submetidas à CB é segura para o binômio materno-fetal. Entretanto, as pacientes fazem } \\
\text { parte de uma amostra selecionada em consultórios particulares, o que pode influenciar } \\
\text { positivamente no resultado da pesquisa. }\end{array}$ \\
\hline
\end{tabular}

REAS/EJCH | Vol.12(2) | e3043 | DOI: https://doi.org/10.25248/reas.e3043.2020 Página 5 de 11 


\begin{tabular}{|c|c|c|c|c|}
\hline $\begin{array}{l}\text { Chevrot A, et } \\
\text { al. } 2016\end{array}$ & Science Direct & $\begin{array}{c}\text { Retrospec } \\
\text { tivo }\end{array}$ & $\begin{array}{l}\text { Avaliar impacto da CB nos } \\
\text { resultados da gravidez e do } \\
\text { tipo de CB no risco } \\
\text { de restrição do crescimento } \\
\text { fetal. }\end{array}$ & $\begin{array}{l}\text { Grupo de estudo constituído por } 139 \text { pacientes, } 58 \text { tiveram um procedimento de má } \\
\text { absorção (by-pass gástrica), } 81 \mathrm{um} \text { procedimento restritivo ( } 72 \text { banda gástrica e } 9 \\
\text { gastrectomia vertical). Comparado com os controles, o grupo de estudo apresentou taxa } \\
\text { reduzida de diabetes gestacional.. }\end{array}$ \\
\hline $\begin{array}{l}\text { Froylich D, et } \\
\text { al. } 2016\end{array}$ & Science Direct & Coorte & $\begin{array}{l}\text { Comparar o efeito da } \\
\text { gravidez antes e após a CB } \\
\text { na perda geral de peso. }\end{array}$ & $\begin{array}{l}\text { Participaram 62, as quais os dados foram comparados com } 92 \text { pacientes que nunca } \\
\text { haviam concebido. A idade média na cirurgia é } 33,8 \text { anos, IMC médio na cirurgia é } 48,2 \\
\mathrm{~kg} / \mathrm{m}^{2} \text {. Após acompanhamento por } 43,9 \text { meses, a perda de excesso de peso é } 68,0 \% \text { no } \\
\text { grupo não gestante e } 53,0 \% \text { no grupo gestante. Análises mostraram que gravidez antes } \\
\text { da CB teve efeito negativo na perda de peso em relação a pacientes que nunca } \\
\text { engravidaram. }\end{array}$ \\
\hline $\begin{array}{l}\text { Makaronidis } \\
\text { J, } \\
\text { et al. } 2017\end{array}$ & $\mathrm{NCBI}$ & Coorte & $\begin{array}{l}\text { Realizar estudo de coorte } \\
\text { retrospectivo de centro } \\
\text { único de gestações em } \\
\text { mulheres pós-CB. }\end{array}$ & $\begin{array}{l}\text { O estudo com } 69 \text { mulheres acompanhadas por } 8 \text { anos, com } 79 \text { gestações e } 18 \\
\text { perdidas. Taxas de cesariana foi semelhante em gestações concebidas antes ou após } \\
\text { primeiro ano pós CB, das quais, } 2 \text { desenvolveram diabetes gestacional }(1,9 \%), 3 \\
\text { hemorragia pós-parto }(2,9 \%) \text {. O peso médio ao nascer foi } 3,11 \mathrm{~kg} \text { e idade gestacional } \\
\text { média ao nascer } 39,5 \text { semanas. Sendo recomendado adiar a concepção até } 1 \text { ano após } \\
\text { a cirurgia }\end{array}$ \\
\hline $\begin{array}{l}\text { Stephansson } \\
\text { O e } \\
\text { Johansson K } \\
\quad 2017\end{array}$ & $\mathrm{NCBI}$ & Coorte & $\begin{array}{l}\text { Relatar o risco de parto } \\
\text { prematuro entre uma coorte } \\
\text { expandida de mulheres que } \\
\text { deram à luz entre } 2006 \text { e } \\
2013 \text {. }\end{array}$ & $\begin{array}{l}\text { Estudo com base populacional } 1.941 \text { nascimentos de mulheres submetidas a CB e } 6.574 \\
\text { nascimentos controle. Total de } 163 \text { de } 1.941 \text { mulheres submetidas à CB tiveram parto } \\
\text { prematuro (<37 semanas completas de gestação) em comparação com } 447 \text { de } 6.574 \text { do } \\
\text { controle. Sendo constatada associação entre história de CB e risco de parto prematuro. }\end{array}$ \\
\hline
\end{tabular}




\begin{tabular}{|c|c|c|c|c|}
\hline $\begin{array}{c}\text { Yau PO, et } \\
\text { al. } \\
2017\end{array}$ & Scielo & $\begin{array}{l}\text { Meta- } \\
\text { análise }\end{array}$ & $\begin{array}{l}\text { Examinar o efeito do tempo } \\
\text { decorrido da cirurgia à } \\
\text { concepção no curso da } \\
\text { gravidez e os resultados } \\
\text { em pacientes bariátricos. }\end{array}$ & $\begin{array}{l}\text { Grupo de } 54 \text { mulheres que engravidaram após CB. Das } 26 \text { das } 41 \text { foram submetidas a } \\
\text { CB menos de } 2 \text { anos antes da concepção e } 15 \text { submetidas há mais de } 2 \text { anos antes da } \\
\text { concepção. Dessas, } 8 \text { sofreram aborto espontâneo. Não houve diferenças entre os } \\
\text { grupos nos partos prematuros, internação UTIN, ganho de peso gestacional, deficiências } \\
\text { nutricionais, diabetes ou hipertensão gestacional. Sendo assim, gestação nos primeiros } \\
2 \text { anos após CB parece não ter efeito no curso e resultado da gravidez. }\end{array}$ \\
\hline $\begin{array}{c}\text { Cunha CPV } \\
2018\end{array}$ & Scielo & $\begin{array}{l}\text { Descritivo } \\
\text { retrospect } \\
\quad \text { ivo }\end{array}$ & $\begin{array}{l}\text { Verificar a relação entre a } \\
\text { adequação nutricional de } \\
\text { grávidas submetidas a CB e } \\
\text { os resultados neonatais. }\end{array}$ & $\begin{array}{l}\text { Participaram } 58 \text { gestantes pós CB. Foi avaliado IMC pré-concepcional, ganho ponderal } \\
\text { gestacional e dados bioquímicos no início e fim da gravidez, relacionando as variáveis e } \\
\text { peso ao nascer do recém-nascido, onde pós CB, IMC pré-concepcional e níveis séricos } \\
\text { maternos de vários nutrientes, triglicerídeos, zinco e ácido fólico foram associados a } \\
\text { alterações do peso. }\end{array}$ \\
\hline $\begin{array}{l}\text { Stephansson } \\
\text { O, et al. } 2018\end{array}$ & Science Direct & $\begin{array}{c}\text { Coorte } \\
\text { prospectiv } \\
0\end{array}$ & $\begin{array}{ll}\text { Investigar o risco } & \text { de } \\
\text { desfechos adversos no } & \text { no } \\
\text { parto entre mulheres no } & \text { nós-operatório } \\
\text { pós em } \\
\text { comparação com mulheres } \\
\text { sem histórico de CB, mas } \\
\text { com características } \\
\text { semelhantes }\end{array}$ & $\begin{array}{l}\text { Foram identificados } 1.929 \text { nascimentos em mulheres com histórico de CB e desfecho } \\
\text { foram modo e indução de parto, gravidez pós-termo ( } \geq 42 \text { semanas de gestação), } \\
\text { analgesia peridural, sofrimento fetal. IMC médio pré-operatório } 42,6 \mathrm{~kg} / \mathrm{m}^{2} \text {, intervalo } \\
\text { médio entre CB e concepção } 1,4 \text { anos e perda média de IMC entre CB e gravidez precoce } \\
13,5 \mathrm{~kg} / \mathrm{m}^{2} \text { ( } 38 \mathrm{~kg} \text { ), que, comparadas ao controle, pacientes pós CB tiveram menor } \\
\text { probabilidade de parto cesáreo. }\end{array}$ \\
\hline $\begin{array}{l}\text { Mengesha } \\
\text { BM, } \\
\text { et al. } 2018\end{array}$ & Science Direct & $\begin{array}{c}\text { Transvers } \\
\text { al }\end{array}$ & $\begin{array}{l}\text { Determinar prevalência de } \\
\text { contraceptivos } \\
\text { perioperatórios e no } \\
\text { intervalo da gravidez entre } \\
\text { mulheres submetidas a CB } \\
\text { e descrever uso de } \\
\text { contraceptivos no pós CB } \\
\text { no primeiro ano de cirurgia } \\
\text { nessa população. }\end{array}$ & $\begin{array}{l}\text { Grupo de } 363 \text { mulheres, idades entre } 18 \text { e } 45 \text { anos submetidas a CB nos últimos } 24 \\
\text { meses, das quais } 66 \% \text { relataram usar contraceptivos nos primeiros } 12 \text { meses pós CB, } \\
27 \% \text { contraceptivos orais e } 26 \% \text { usavam aparelho intra - uterino. Sendo assim, mulheres } \\
\text { em idade reprodutiva devem ser aconselhadas no período perioperatório sobre gravidez } \\
\text { e contracepção, para que possam tomar decisões sobre prevenção da gravidez } \\
\text { perioperatória e uso de métodos contraceptivos. }\end{array}$ \\
\hline
\end{tabular}

Fonte: Rosales JJB, et al., 2020.

REAS/EJCH | Vol.12(2) | e3043 | DOI: https://doi.org/10.25248/reas.e3043.2020 Página 7 de 11 
Com relação ao ano de publicação, como apresentado no Quadro 1, o ano de 2016 concentrou maior número de artigos utilizados, quatro, seguido pelos anos de 2018 e 2017, com três publicações cada um e do ano de 2015 foram utilizadas duas publicações. Com relação ao tipo de estudo, todos os artigos são oriundos de pesquisas originais, realizadas diretamente com pacientes ou bancos de informações, são estudos do tipo coorte, coorte retrospectivo e prospectivo, meta-análise, retrospectivo, descritivo retrospectivo e transversal. Os periódicos de origem dos artigos, grande parte são da área da medicina e saúde, com artigos nacionais e internacionais, reafirmando mais uma vez, a relevância da temática em estudo e do rigor metodológico que os artigos se encontram.

\section{DISCUSSÃO}

A prevalência da obesidade vem crescendo de forma exponencial nos últimos anos em todo o mundo, tornando-se um problema de saúde pública, devido ao consumo excessivo de carboidratos, alimentos processados, elevado teor de açúcares simples presente na alimentação, gorduras e elevada taxa de sedentarismo. Por ser um problema mundial, assim como em todos os indivíduos, as mulheres grávidas, as quais apresentam sobrepeso ou estão em estado de obesidade, apresentam grandes riscos de complicações durante o período gestacional (WHO, 2016).

Para gestantes com sobrepeso ou obesas, o risco de complicações durante o período gestacional é grande, tais como diabetes gestacional, pré-eclampsia, macrossomia fetal e hemorragia pós-parto. Filhos cuja mães apresentam estado de obesidade tem grandes chances de desenvolver doenças que afetam a saúde durante a vida toda como hipertensão, diabetes ou doenças cardiovasculares, por alterações epigenéticas associadas ao estado materno. Sendo assim, a CB apresenta - se como o método mais efetivo na prevenção dessas complicações (BEST D, et al., 2017; CATALANO PM e SHANKAR K, 2017; FALCONE V, et al., 2018).

A CB, por ser a forma mais eficaz de perda de peso a longo prazo, é um dos principais procedimentos utilizados atualmente, sendo mais da metade das cirurgias realizadas no mundo, em mulheres em idade fértil. Pacientes submetidas a CB antes da gestação apresentam menor predisposição a comorbidade associadas a obesidade, tais como diabetes gestacional e hipertensão, entretanto, estão mais suscetíveis a deficiências de micronutrientes, como deficiência em ferro e vitamina $D$, as quais estão relacionadas com o parto prematuro e defeitos no tubo neural do feto (AKHNTER Z, et al., 2019).

Dentre os procedimentos mais frequentes dentro da CB, a Gastrectomia Vertical (GV) é uma das operações mais populares e regularmente executada, onde a maior curvatura do estômago é ressecada, reduzindo assim, o volume do estômago em, aproximadamente, $75 \%$, o que limita a ingestão alimentar, além de remover células endócrinas secretoras, as quais produzem grelina, auxiliando na redução do apetite. Tal procedimento auxilia na melhora da homeostase da glicose, auxiliando na remissão do diabetes (SHAWE J, et al., 2017; AKHNTER Z, et al., 2019).

O Bypass Gástrico em Y de Roux (RYGB) é um procedimento o qual o volume do estômago é reduzido para, aproximadamente 15 a $30 \mathrm{ml}$, sendo a absorção nutricional prejudicada, com fluxo alimentar desviado para a porção distal do intestino delgado. Tal procedimento induz uma limitada ingestão oral, má absorção alimentar, porém, esse último apresenta redução com o tempo, devido ao processo de hipertrofia intestinal. Tal procedimento estimula o aumento da secreção hormonal Glucagon-like-peptide-1 (GLP-1) e Peptídeo YY (PYY) o intestino, o que leva a diminuição do apetite e homeostase da glicose (SHAWE J, et al., 2017; AKHNTER Z, et al., 2019).

A Banda Gástrica Ajustável (BGA) consiste na inserção de uma banda restritiva inflável ao redor do estômago, assim criando uma pequena bolsa, a qual apresenta uma abertura estreita para a porção inferior do estômago, sendo ajustada por adição ou remoção do fluído por meio de um orifício subdérmico, reduzindo o apetite e a capacidade de armazenamento de alimentos no estômago. Outros procedimentos cirúrgicos incluem desvio biliopancreático com interruptor duodenal, balão intragástrico, porém tais procedimentos apresentam - se desatualizados ou raramente realizados (SHAWE J, et al., 2017; AKHNTER Z, et al., 2019). 
Segundo Stephansson O, et al. (2018), é comprovado que a perda de peso tem a capacidade de reduzir adversidades em resultados maternos e perinatais, sendo assim, é passível de suposição que a CB tem a capacidade de alcançar os mesmos resultados positivos. Deve-se, entretanto, estar ciente que a CB pode aumentar o risco de deficiências nutricionais, partos prematuros e partos prematuros espontâneos, desfechos gestacionais com complicações e problemas para mãe e filhos devendo a paciente saber quais as consequências o procedimento pode ter sobre a mesma e sobre o feto.

As diretrizes estabelecidas na atualidade têm como recomendação, que a gestação seja adiada por um período que corresponde entre 12 a 24 meses, aproximadamente, após o procedimento de CB, com a finalidade de permitir estabilização do peso corporal, bem como identificação de procedimentos e tratamentos adequados para suprir possíveis deficiências nutricionais da paciente. Entretanto, tais recomendações devem ser feitas de formas individualizada, levando - se em consideração parâmetros antropométricos e clínicos específicos de cada paciente (GONZÁLES I, et al., 2016; MAKARONIDIS J, et al., 2017; FALCONE V, et al., 2018).

Yau PO, et al. (2017) e Gonzáles I, et al (2016) afirmam que em seus estudos que a concepção/ gravidez 24 meses após o procedimento de CB não apresentaram efeitos negativos no curso e desfecho gestacional. Os estudos, em seus achados, não revelaram aumento nas taxas das complicações como risco de nascimento prematuro, admissão na Unidade de Terapia Intensiva Neonatal (UTIN), deficiências nutricionais, hipertensão e diabetes gestacional nas pacientes e filhos. Porém, não existem evidências científicas, nem consenso que possam definir, como padrão, o período de espera para a gestação pós CB.

É de conhecimento mundial que a obesidade está relacionada a inúmeras comorbidades, tais como hipertensão, distúrbios músculo - esqueléticos, hipertensão e diabetes tipo 2. Com isso, as gestantes com sobrepeso ou obesidade apresentam um risco bastante elevado de diabetes gestacional, aborto espontâneo, parto prematuro, pré - eclampsia e hemorragia pós-parto. Já os filhos podem apresentar alterações fetais, como disfunções neurológicas, malformações cardiovasculares, espinha bífida e morbimortalidade neonatal (FALCONE V, et al., 2018).

Atualmente não existe triagem específica para detecção e tratamento para diabetes gestacional em mulheres pós $\mathrm{CB}$, porém, as submetidas ao procedimento apresentam menor risco de desenvolver diabetes tipo 2 e/ou gestacional em relação as que não foram submetidas a CB. $O$ diabetes não diagnosticado durante a gestação pode resultar em grandes riscos de anomalias fetais e outros desfechos adversos. A triagem para detecção do diabetes gestacional deve ser realizada dosando a glicose plasmática em jejum (FPG), $\geq 65 \%$, e Hba1c, $\geq 7,0 \mathrm{mmol} / \mathrm{L}$, de acordo com os dados da American Diabetes Association, devido a inexistência de valores para o período gestacional pós CB (CHEVROT A, et al., 2016; SHAWE J, et al., 2017).

Além da diabetes gestacional, a hipertensão gestacional é uma das comorbidades que mais afetam mulheres nesse período, contribuindo para aumento de duas a três vezes o risco de pré - eclampsia, principalmente em pacientes acima do peso. Para pacientes submetidas a $\mathrm{CB}$, a hipertensão gestacional não tem uma prevalência definida, porém, hipótese afirmam que as chances de mulheres em período gestacional pós $\mathrm{CB}$ apresentam chances de duas a três vezes menor de apresentar distúrbios hipertensivos associados a gravidez (GONZÁLES I, et al., 2016).

Sobre perda fetal e morte perinatal, sabe - se que abortos espontâneos ocorrerem em cerca de $10 \%$ a $20 \%$ das gestações gerais, sendo a obesidade caracterizada como dos fatores de risco. Em pacientes submetidas a CB, é observado um aumento da mortalidade intrauterina e neonatal, assim como a taxa de abortos espontâneos, quando comparadas com a população geral. Tal resultado deve-se a fatores como má absorção alimentar da mãe, que, por não receber suprimentos nutricionais suficientes para ela e o filho, acabam por induzir a perda fetal ou até mesmo a morte perinatal (JOHANSSON K, et al., 2015; SHAWE J et al., 2017).

Pouco se relata sobre prevalência de complicações intraparto em gestantes pós CB. O parto prematuro e o parto prematuro espontâneo, como afirma Johansson K, et al (2015) e Stephansson O e Johansson K (2017) apresenta taxa mais elevada em pacientes que passaram por $\mathrm{CB}$, devido a comorbidades associadas 
envolvidas. As taxas e partos cesáreos apresentam valores variáveis, sem diferenças entre pacientes submetidas ao procedimento em relação aos que não foram submetidas. Apesar de resultados sem confirmação, não existem fatores fisiopatológicos que afirmem que cesarianas possam ser mais frequentes em pacientes submetidas a CB (JOHANSSON K, et al., 2018).

Análises nutricionais em pacientes pós $C B$ mostram que as mesmas dispõem de riscos elevado em desenvolver deficiências em absorção de micronutrientes, e em gestantes pós $C B$, existe carência de evidências sobre as estratégias de monitoramentos e suplementação nutricional. Akhnter Z, et al. (2019) e Shawe J, et al. (2017) recomendam que a gravidez seja planejada, e a suplementação nutricional seja otimizada, de forma preferencial, de 3 a 6 meses antes da concepção. Deve - se administras um suplemento multivitamínico e mineral diariamente, antes e durante o período gestacional, que deve conter cobre (2 mg),

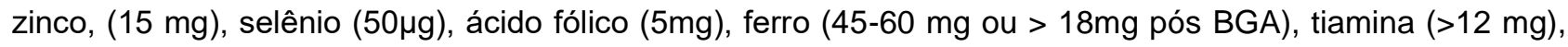
vitamina $\mathrm{E}(15 \mathrm{mg})$ e vitamina $\mathrm{A}$ (5000 UI). Essa suplementação deve ser ajustada para se manter as concentrações dentro dos limites normais da paciente.

Mundialmente, existem diretrizes para uma variedade de grupos relacionados a gravidez de alto risco, como diabetes, hipertensão e obesidade. O estudo de Akhnter Z, et al. (2019) afirmam que as pacientes pós $\mathrm{CB}$ que engravidam constituem um grupo de risco elevado, sendo necessário o desenvolvimento de diretrizes para profissionais de saúde, à medida que a obesidade e a $\mathrm{CB}$ aumentam sua frequência cada vez mais. $\mathrm{O}$ embasamento em evidências e protocolos atuais podem ser usados para informar e aumentar a comunicação sobre os riscos e formas de prevenções em futuras gestações de mulheres em idade gestacional antes e pós CB.

Para aquelas pacientes que já apresentam um histórico de $\mathrm{CB}$, é necessário um acompanhamento fixo nutricional na preconcepção, bem como aumento no monitoramento do feto, avaliação nutricional e acompanhamento glicêmico durante todo o período gestacional. Entretanto, mais estudos são necessários para definir qual das formas de intervenção cirúrgica, a cirurgia restritiva ou a cirurgia mal absortiva, resultam em melhores resultados perinatais, sem comprometimento dos resultados maternos, promovendo o bemestar de mãe e filho (AKHNTER Z, et al., 2019).

\section{CONSIDERAÇÕES FINAIS}

É perceptível o crescimento de procedimentos de CB em mulheres, principalmente aquelas que se encontram em idade fértil, entretanto ainda não existe consenso internacional sobre manejo da gravidez pós $\mathrm{CB}$, entretanto, é considerada segura tanto para mãe quanto para o feto, levando - se em consideração e aguardando o período de espera entre a CB e a gravidez, assim como as particularidades físicas e clínicas da paciente. Não foram encontradas diferenças significativas nos desfechos maternos e fetais entre gestações ocorridas antes ou depois de 24 meses pós CB. Entretanto, mulheres que engravidam após CB apresentam menor risco de apresentar diabetes gestacional, distúrbios hipertensivos, e maior risco de deficiências nutricionais maternas, recém-nascido com baixo peso ao nascer, restrição de crescimento intrauterino, partos prematuros e mortalidade intrauterina e neonatal.

\section{REFERÊNCIAS}

1. BEST D, AVENELL A, BHATTACHARYA S. Qual a eficácia das intervenções para perda de peso para melhorar a fertilidade em mulheres e homens com sobrepeso ou obesidade? Uma revisão sistemática e metanálise das evidências. Hum Reprod Update. 2017; 23 (6): 681-705.

2. BUSETTO L, et al. Practical recommendations of the Obesity management task force of the European Association for the Study of Obesity for the post-Bariatric Surgery medical management. Obes Facts. 2018;10(6):597-632.

3. CATALANO PM, SHANKAR K. Obesidade e gravidez: mecanismos de consequências adversas a curto e longo prazo para mãe e filho. BMJ. 2017; 356: j1.

4. CHEVROT A, et al. Impact of bariatric surgery on fetal growth restriction: experience of a perinatal and bariatric surgery center. American Journal of Obstetrics and Gynecology. 2016; v. 214, Issue 5; 655e.1-655.e7. 
5. CUNHA CPV. Gravidez após cirurgia bariátrica: relação entre a adequação nutricional da grávida e os resultados neonatais. Trabalho de investigação, Centro Hospitalar do Porto, E. P. E. 1.ํ Ciclo em Ciências da Nutrição Faculdade de Ciências da Nutrição e Alimentação da Universidade do Porto Porto, 2018; 27 p.

6. FALCONE V, et al. Pregnancy after bariatric surgery: a narrative literatura review and discussion of impacto $n$ pregnancy management and outcome. BMC Pregnancy and Childbirth. 2018, 507.

7. FROYLICH D, et al. The effect os pregnancy before and/or after bariatric surgery on weight loss. Surgery for Obesity and Related Diseases. 2016; v 12, Issue 3. p. 596-599.

8. GONZALES I, et al. Pregnancy after bariatric surgery: improving outcomes for mother and child. International Journal of womens's Health. 2016, 8: 721-729.

9. incorporação de evidências na saúde e na enfermagem. Texto contexto enferm., 2008; 17(4): 758-764.

10. JOHANSSON K, et al. Outcomes of pregnancy after bariatric surgery. N Engl J Med. 2015, 372:814-824.

11. KIZY S, et al. National trends in bariatric surgery 2012-2015: demographics, procedure selection, readmissions, and cost. Obes Surg. 2017; 27(11): 2933- 2939.

12. KWONG W, et al. Maternal and neonatal outcomes after bariatric surgery: a systematic review and meta-analysis the benefits outweigh the risks? American Journal of Obstetrics and Gynecology. 2018, v 218, p. 573-580.

13. MAKARONIDIS J, et al. Pregnancy after bariatric surgery: a single-centre retrospective cohort study. Society for Endocrinology. 2017.

14. MARQUES RR, MARQUES RR, MARQUES I. gestação após cirurgia bariátrica: série de casos e revisão de literatura. 2016, Unoesc \& Ciência - ACBS Joaçaba, v. 7, n. 1, p. 69-76

15. MENDES KDS, SILVEIRA RCCP, GALVÃO CM. Revisão integrativa: método de pesquisa para a

16. MENGESHA BM, et al. Perioperative pregnancy interval contraceptive counseling experiences, and contraceptive use in women undergoing bariatric surgery. American Journal os Obstetrics and Gynecology. 2018; v 219. Issue 1, 81. E1-81. E9.

17. Obesity and overweight. World Health Organization. Disponível em: http://www.who.int/news-room/factsheets/detail/obesity-and-overweight. Acesso em: 10 nov. 2019.

18. PARKER MH, BERGUELLA V, NIJJAR JB. Bariatric surgery and associated adverse pregnancy outcimes among obese women. The Journal of Maternal-Fetal \& Neonatal Medicine. 2016, v 29, Issue 11.

19. SHAWE J, et al. Pregnancy after bariatric surgery: Consensus recommendations for periconception, antenatal and postnatal care. Obesity Reviews. 2019, v 20, ISSUE 11.

20. STEPHANSSON O, et al. Delivery outcomes in term births after bariatric surgery: Population-based matched cohort study. PLoS Med. 2018;15 (9): e10022656.

21. STEPHANSSON O, JOHANSSON K. Bariatric surgery and preterm birth. N Engl J Med. 2016;375(8):805-806.

22. YAU PO, et al. Pregnancy after bariatric surgery: the effect of time-to-conception on pregnancy outcomes. Surgery for Obesity and Related Diseases. 2017, v 13, Issue 11, 1899-1905 p. 